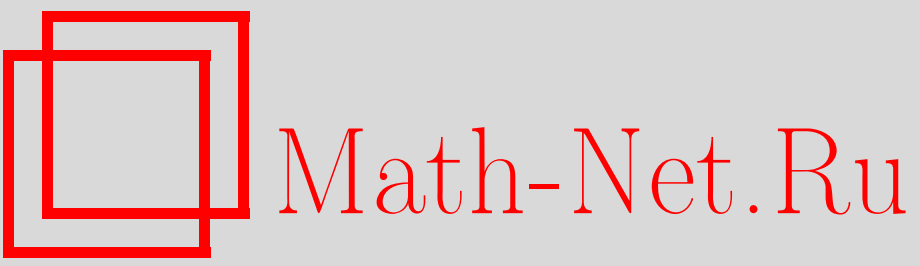

А. В. Забродин, Иерархия Уизема в задачах роста, ТМФ, 2005, том 142, номер 2, 197-217

DOI: https://doi.org/10.4213/tmf1776

Использование Общероссийского математического портала Math-Net.Ru подразумевает, что вы прочитали и согласны с пользовательским соглашением

http://www.mathnet.ru/rus/agreement

Параметры загрузки:

IP: 18.208 .226 .222

26 апреля 2023 г., 09:59:13 
ТЕОРЕТИЧЕСКАЯ

И МАТЕМАТИЧЕСКАЯ

ФИЗИКА

Том 142, № 2

февраль, 2005

(C) 2005 г.

А.В. Забродин ${ }^{*}$

\section{ИЕРАРХИЯ УИЗЕМА В ЗАДАЧАХ РОСТА}

Обсуждается недавно установленная эквивалентность между лапласовским ростом в пределе нулевого поверхностного натяжения и универсальной иерархией Уизема, известной в теории солитонов. Эта эквивалентность позволяет выделить класс точных решений задачи о лапласовском росте в многосвязном случае. Эти решения соответствуют конечномерным редукциям иерархии Уизема, которые могут быть представлены как уравнения гидродинамического типа, разрешимые с помощью обобщенного метода годографа.

Ключевые слова: проблема Саффмана-Тейлора, лапласовский рост, уравнения Уизема, функция Шварца.

\section{1. ВВЕДЕНИЕ}

Динамика движушегося фронта (гранищы раздела между двумя различными фазами), вызванная гармоническим скалярным полем, часто возникает в разнообразных физических и математических контекстах и имеет ряд важных практических приложений. Процессы такого типа обобщенно называются лапласовским ростом. Их важнейшая общая черта - наличие гармонического поля, которое служит потенциалом для поля скоростей роста.

Наиболее активно динамика границы раздела изучалась в случае двумерной пространственной геометрии. Для определенности мы будем говорить о границе раздела между двумя несмешиваюшимися и несжимаемыми жидкостями с сильно различающимися вязкостями на плоскости. На практике двумерная геометрия реализуется в узкой щели между двумя параллельными стеклянными пластинами (ячейка Хеле-Шоу). Эта версия задачи называется также проблемой Сафффмана-Тейлора (см. обзор [1]).

Рассмотрим компактную плоскую область, занятую жидкостью с пренебрежимо малой вязкостью (водой). Будем называть ее водяной каплей. Пусть ее внешность занята вязкой жидкостью (нефтью). Предположим, что граница раздела между нефтью и водой представляет собой гладкий замкнутый контур. Нефоть равномерно откачивается

${ }^{*}$ Институт биохимической физики РАН, Институт теоретической и экспериментальной физики, Москва, Россия. E-mail: zabrodin@itep.ru 
через сток (насос), находящийся в некоторой фиксированной точке (возможно, на бесконечности), а в водяную каплю при этом поступает вода. В более общем случае можно рассмотреть несколько неф̆тяных насосов и несколько несвязных водяных капель. В этой обшей постановке задача о лапласовском росте обсуждалась в работах [2]-[4].

В области, занятой нефтью, локальная скорость жидкости $\vec{V}=\left(V_{x}, V_{y}\right)$ пропорциональна градиенту давления $p=p(x, y)$ (закон Дарси):

$$
\vec{V}=-\kappa \nabla p
$$

где $\kappa$ называется коэффициентом фильтрации. Он обратно пропорционален вязкости жидкости. Для удобства дальнейшего изложения мы выберем единицы измерения таким образом, чтобы в нефти $\kappa=1 / 4$. Закон Дарси имеет место, в частности, на внешней поверхности границы раздела, определяя ее динамику: $V_{n}=-\partial_{n} p / 4$. Здесь $V_{n}-$ скорость роста, направленная по определению вдоль нормали к границе раздела, $\partial_{n}-$ нормальная производная. Так как обе жидкости несжимаемы $(\nabla \vec{V}=0)$, закон Дарси означает, что давление $p$ является гармонической функцией во внешности (в нефти) везде, кроме точки, в которой находится насос. В этой точке имеется логарифмическая особенность.

Таким образом, поле давления является решением зависяшей от времени краевой задачи для оператора Лапласа $\Delta=\partial_{x}^{2}+\partial_{y}^{2}$ с заданной логарифмической особенностью и определенным условием на гранище растущей области. Это условие фиксируется из следующих соображений. Если вязкость воды достаточно мала, внутри водяных капель давление однородно ( $p=$ const). Мы будем предполагать, что давление не имеет скачка на границе раздела, так что функция $p(x, y)$ постоянна вдоль нее. Это означает, что поверхностное натяжение считается равным нулю. Итак, математическая формулировка задачи лапласовского роста в пределе нулевого поверхностного натяжения имеет следуюший вид:

$$
\begin{aligned}
& V_{n}=-\frac{1}{4} \partial_{n} p, \\
& \Delta p=0 \text { в нефти, причем } d p=0 \text { вдоль границы, } \\
& p(x, y)=\ln \rho^{2}+\cdots \quad \text { при } \rho \rightarrow 0 .
\end{aligned}
$$

Здесь $\rho$ - расстояние между стоком и точкой $x, y$. Если сток находится на бесконечности, что часто предполагается во внешней задаче роста, последнее условие заменяется асимптотикой $p=-\ln \left(x^{2}+y^{2}\right)+\cdots$ на большом расстоянии от капли. Если область, занятая нефтью, многосвязна (т.е. если имеется более чем одна капля воды), эта формулировка должна быть дополнена некоторыми физическими условиями на давление в каплях воды, которые мы не будем здесь обсуждать. Задача заключается в нахождении эволюции гранищы раздела между жидкостями с течением времени.

В односвязном случае (одна капля воды) эффективным оказывается применение техники конформных отображений (см., например, [1], [3]). Перейдя к комплексным координатам $z=x+i y, \bar{z}=x-i y$, можно описать процесс роста с помошью зависяшего от 
времени конформного отображения $z=f(w, T)$ фиксированной области простой формы (например, единичного диска) на эволюционируюшую область, занятую нефтью. Граница раздела является образом единичного круга $w=e^{i \phi}, 0 \leqslant \phi \leqslant 2 \pi$. Уравнения динамики (1) переходят при этом в нелинейное дифференциальное уравнение в частных производных на функцию $f(w, T)$, которое называют уравнением лапласовского роста. Если сток нефти находится на бесконечности, оно имеет вид

$$
2 \operatorname{Im}\left(\frac{\partial f\left(e^{i \phi}, T\right)}{\partial \phi} \frac{\overline{\partial f\left(e^{i \phi}, T\right)}}{\partial T}\right)=1
$$

Это уравнение впервые появилось в 1945г. в работах [5], посвяшенных математическим вопросам теории добычи нефти.

Позднее были найдены некоторые точные конечномерные редукции уравнения лапласовского роста. В предположении, что производная конформного отображения $f$ рациональная функция, это уравнение с помошью прямой подстановки сводится к конечномерной динамической системе относительно параметров этой функции (скажем, ее полюсов, нулей или вычетов). Различные варианты таких динамических систем содержатся в [6], [7]. Оказывается, что эти системы в действительности могут быть проинтегрированы в том смысле, что все производные по времени можно устранить. В результате полюсы функции $f$ записываются как неявные функции времени.

Сушествование конечномерных редукций послужило серьезной мотивацией для поиска интегрируемой структуры, лежашей в основе модели. В случае одной капли воды эта структура была отождествлена в [8] с двумеризованной иерархией Тоды в пределе нулевой дисперсии. Уравнение лапласовского роста - это струнное уравнение для иерархии Тоды. Конформное отображение играет роль функции Лакса.

Векторное поле $\partial / \partial T$ в пространстве областей задает определенный поток в иерархии Тоды. Если откачка нефти происходит с помощью единственного насоса, другие потоки “заморожены". Чтобы их "разморозить”, надо разрешить откачивать нефть из любой точки на плоскости. В обшем случае имеется столько разных потоков, сколько может быть различных типов и местоположений нефьяных насосов. Удобно ассоциировать с каждым насосом собственную временну́ю переменную, которая равна полному количеству нефти, откачанной данным насосом. Известно [4], что результат эволюции при лапласовском росте в случае нескольких насосов зависит только от полного количества неф̆ти, откачанной каждым из них, и не зависит от порядка их работы. В контексте теории интегрируемых систем этот факт отражает коммутативность потоков в иерархии Тоды.

Недавно этот подход был обобшен [9] на лапласовский рост нескольких водяных капель. В этом случае динамика оказывается эквивалентной универсальной иерархии уравнений Уизема на пространстве модулей римановых поверхностей рода $g$, где $g$ на единицу меньше числа капель воды. В полной общности эта иерархия была введена Кричевером [10], [11] в связи с теорией медленных модуляций точных периодических решений солитонных уравнений. Бездисперсионная иерархия Тоды - ее простейший частный 
случай, отвечающий $g=0$. Известно, что универсальная иерархия Уизема имеет большое количество содержательных конечномерных редукций при любом $g$. Они представляют собой системы уравнений гидродинамического типа, которые допускают неявное решение с помощью обобщенного метода годографа [12]. Применив эти результатык проблеме лапласовского роста, мы получаем обширное семейство решений для многосвязного случая. Бесконечномерная контурная динамика для них сводится к конечным системам дифференциальных уравнений, которые могут быть проинтегрированы.

Цель данной статьи - изучить этот класс конечномерных редукций и прояснить их алгебро-геометрический смысл.

\section{2. ЛАПЛАСОВСКИЕ ПОТОКИ И УНИВЕРСАЛЬНАЯ ИЕРАРХИЯ УИЗЕМА}

В этом разделе объясняется точный смысл утверждения, что лапласовский рост описывается уравнениями Уизема.

Мы используем следующие обозначения (рис. 1). Пусть $\mathrm{D}_{\text {oil }}$ - область плоскости, занятая нефьью (некомпактная область, содержащая бесконечность), а D - область, занятая водой. Мы будем предполагать, что имеется $g+1$ капель воды, которые представляют собой компактные области, ограниченные гладкими непересекающимися замкнутыми контурами $\gamma_{\alpha}, \alpha=0,1, \ldots, g$. Пусть $\mathrm{D}_{\alpha}-\alpha$-я капля воды, так что $\mathrm{D}$ является объединением всех $\mathrm{D}_{\alpha}$. Через $\gamma$ обозначим совокупность контуров $\gamma_{\alpha}: \gamma=\bigcup_{\alpha=0}^{g} \gamma_{\alpha}$. B наших предположениях ни один контур $\gamma_{\alpha}$ не лежит внутри другого.

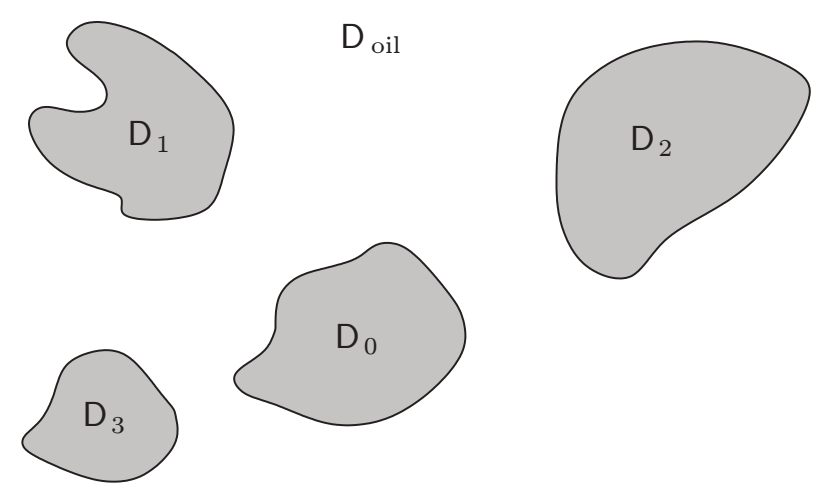

Рис. 1. Капли воды $\mathrm{D}_{\alpha}(g=3)$.

2.1. Коммутативность лапласовских потоков. Любой процесс роста задает поток в пространстве контуров ${ }^{1)}$, или (в инфинитезимальном варианте) векторное поле.

\footnotetext{
1) В зависимости от конкретной задачи поток может быть определен только на подходящем подмногообразии или на некоторой подобласти в пространстве контуров, но это ограничение для нас здесь не существенно.
} 
Его интегральные кривые - это однопараметрические семейства контуров $\gamma(T)$, полученных из произвольного начального контура $\gamma(0)$ в результате эволюции за время $T$.

Потоки, соответствующие лапласовскому росту (лапласовские потоки), задаются положением нефтяных насосов. Именно, пусть насос находится в точке $a \in \mathrm{D}_{\text {оil }}$; тогда решение краевой задачи, заданной в области $\mathrm{D}_{\text {oil }}$,

$$
\Delta p(z)=4 \pi \delta^{(2)}(z-a),\left.\quad d p\right|_{\gamma}=0
$$

(здесь $\delta^{(2)}(z-a)$ означает двумерную дельта-функцию с носителем в точке $a$ с некоторыми дополнительными условиями на границах областей $\mathrm{D}_{\alpha}$ в случае более чем одной капли воды [9]) однозначно задает бесконечно малую деформацию кривой $\gamma$, для которой вектор нормальной скорости равен $-\partial_{n} p / 4$. Такая деформация является касательным вектором к пространству контуров. Для краткости мы в этой статье не обсуждаем другие типы лапласовских потоков из числа рассмотренных в работе [9].

Пусть задан некоторый лапласовский поток; в качестве величины, параметризующей смещение вдоль его траекторий в пространстве контуров, естественно взять количество нефти $T$, откачанное насосом, которым определяется этот поток (если мощность откачки постоянна, $T$ можно отождествить с временем). Так как обе жидкости предполагаются несжимаемыми, $T$ - это также изменение суммарной площади водяных капель. Пусть $\partial_{T}$ - соответствующее векторное поле. Оно определено инвариантным образом, т.е. не зависит от выбора локальных координат в пространстве контуров.

Как обычно, мы отождествим векторные поля с производными вдоль них. Производная любого функционала $F$ на пространстве контуров вдоль векторного поля (производная Ли) определяется следуюшим образом:

$$
\partial_{T} F=\lim _{\epsilon \rightarrow 0} \frac{F(\gamma(T+\epsilon))-F(\gamma(T))}{\epsilon} .
$$

Если имеется несколько стоков (в точках $a, b$ и т.д.), соответствующие векторные поля будут обозначаться $\partial_{T^{(a)}}, \partial_{T^{(b)}}$ и т.д.

Эти обозначения оправдываются следуюшей теоремой [4].

Теорема 1. Векторные поля $\partial_{T^{(a)}}, \partial_{T^{(b)}}$ коммутируют для всех $a, b \in \mathrm{D}_{\text {oil }}$.

Это инфинитезимальная формулировка того факта, что результат эволюции при лапласовском росте (т.е. форма области $\mathrm{D}_{\text {оil }}$ ) зависит только от объемов нефти $T^{(a)}, T^{(b)}$, откачанных из точек $a, b$, и не зависит от прочих деталей этого процесса (в предположении, что не происходит топологической перестройки области и она не становится сингулярной). В свою очередь, этот факт следует из сведения задачи лапласовского роста к обратной задаче теории потенциала в двух измерениях и локальной единственности решения последней. Более подробно этот вопрос обсуждается в [4].

Теорема 1 говорит о том, что $\partial_{T^{(a)}}$ можно считать частной производной в некотором пространстве с координатами $T^{(a)}$. Приведем простой пример такого пространства. Зафиксируем $N$ точек $a_{j}$ и рассмотрим многообразие контуров, которые можно получить 
из некоторой начальной конфигурации в результате всевозможных процессов лапласовского роста с насосами в точках $a_{j}$. Форма области однозначно определяется (если она не становится сингулярной) объемом нефти, откачанной из каждой точки $a_{j}$, т.е. величинами $T^{\left(a_{j}\right)}$. Это конфигурационное пространство $N$-мерно, и $T^{\left(a_{j}\right)}$ являются локальными координатами в нем.

2.2. Дубль Шоттки. Наша следуюшая задача - перенести лапласовские потоки на компактные римановы поверхности с вешественной структурой. Для этого нужна конструкция дубля Шоттки [13], которую мы здесь напомним.

$\mathrm{C}$ областью $\mathrm{D}_{\text {oil }}$ (как и с любой плоской областью с границей) можно канонически ассоциировать ее дубль Шоттки

$$
\Sigma=\mathrm{D}_{\mathrm{oil}} \cup \gamma \cup \mathrm{D}_{\mathrm{oil}}^{*}
$$

где $\mathrm{D}_{\text {oil }}^{*}$ - другая копия области $\mathrm{D}_{\text {oil }}$ (ее “обратная сторона”), приклеенная к ней вдоль гранишы $\gamma$. В дальнейшем мы будем называть $\mathrm{D}_{\text {oil }}$ и $\mathrm{D}_{\text {oil }}^{*}$, соответственно, лииевой и обратной стороной дубля. Говоря формально, точка $P \in \Sigma-$ это пара $P=(z, \sigma)$, где $z \in \mathrm{D}_{\mathrm{oil}}$, a $\sigma= \pm$ указывает на принадлежность точки лицевой или обратной стороне. Если $z \in \gamma$, пары $(z, \pm)$ представляют одну и ту же точку. Имеется естественная проекция $\Sigma \rightarrow \mathrm{D}_{\text {оil }}$, которая переводит точку $P=(z, \sigma) \in \Sigma$ в точку $z \in \mathrm{D}_{\text {oil }}$. В дальнейшем мы иногда пишем $z$ вместо $(z,+)$ и $z^{*}$ вместо $(z,-)$.

После добавления бесконечно удаленной точки для каждой копии $\mathrm{D}_{\text {oil }}$ дубль Шоттки $\Sigma$ становится компактной поверхностью с естественной комплексной структурой. Комплексная структура на лицевой стороне такая же, как у $\mathrm{D}_{\text {оil }}$, а обратная сторона имеет противоположную комплексную структуру. Другими словами, голоморфной координатой в $\mathrm{D}_{\text {оil }}$ является $z$, а в $\mathrm{D}_{\text {oil }}^{*}$ - координата $\bar{z}$. Таким образом, $\Sigma$ - компактная риманова поверхность с антиголоморфной инволюцией $P \mapsto P^{*}$, которая переводит $(z, \sigma)$ в $(z,-\sigma)$. Две стороны дубля являются в некотором смысле зеркальными образами друг друга, так что мы будем назьвать точки, переходящие друг в друга при инволюции, зеркально-симметричными точками. Границы капель - неподвижные точки инволюции. Схематическое изображение дубля Шоттки дано на рис. 2.

Если контуры $\gamma_{\alpha}$ гладкие, дубль является по построению гладкой римановой поверхностью рода $g$. Действительно, каждая его точка, включая точки на гранище $\mathrm{D}_{\text {оil }}$, имеет окрестность в форме диска.

Отметим, что множество неподвижных точек инволюции представляет собой в точности $g+1$ замкнутых контуров, и это число контуров в вешественном сечении максимально возможно для поверхностей рода $g$. Римановы поверхности с антиголоморфной инволюцией, обладающие этим свойством, называются вещественными.

В дальнейшем предполагается, что на дубле фиксирован канонический базис а- и b-циклов. Это можно сделать многими способами. Например, в качестве циклов $a_{\alpha}$ можно взять границы капель $\mathrm{D}_{\alpha}, \alpha=1, \ldots, g$, а в качестве циклов $\mathrm{b}_{\alpha}$ взять некоторые 
пути, соединяющие границу 0-й капли с границей $\alpha$-й капли по лицевой стороне и приходяшие назад по обратной стороне дубля. Каждый выбор базиса циклов соответствует определенным физическим условиям в каплях воды, которые подробно обсуждаются в [9]. Для наших целей в данной работе достаточно предполагать, что базис циклов зафиксирован тем или иным способом.

Любая пара мероморфных функций $\varphi, \tilde{\varphi}$ (которые определены в $\mathrm{D}_{\text {оil }}$ и в комплексно-сопряженной области, соответственно) таких, что $\varphi(z)=\tilde{\varphi}(\bar{z})$ на границе, задает мероморфную функцию на дубле. Именно, эта функция принимает значение $\varphi(z)$ в точке $z$ на лицевой стороне и значение $\tilde{\varphi}(\bar{z})$ в зеркально-симметричной точке $z^{*}$ на обратной стороне. Аналогично мероморфньй дифференциал на дубле представляется парой мероморфных дифференциалов $\varphi(z) d z$ и $\tilde{\varphi}(\bar{z}) d \bar{z}$ таких, что $\varphi(z) d z=\tilde{\varphi}(\bar{z}) d \bar{z}$ вдоль граничных контуров.

Если мероморфный дифференциал $d \omega(z)$ в $\mathrm{D}_{\text {оil }}$ является чисто мнимым на границе, его аналитическое продолжение на противоположную сторону дубля особенно просто. Действительно, в соответствии с принципом отражения Шварца мероморфное продолжение дифференциала на обратную сторону дубля есть $-\overline{d \omega(z)}$. Поэтому для любого полюса такого глобально определенного дифференциала имеется зеркально-симметричный полюс на противоположной стороне. В этом случае мы будем называть $d \omega$ cuмметричным дифференциалом на $\Sigma$.

2.3. Абелевы дифференциалы на дубле. Каждому насосу мы поставим в соответствие абелев дифференциал на $\Sigma$. На лицевой стороне это будет дифференциал $\partial_{z} p d z$, где $p$ - поле давления, создаваемое насосом в вязкой жидкости. Поскольку $d p=$ $\partial_{z} p d z+\partial_{\bar{z}} p d \bar{z}=0$ вдоль границы, $\partial_{z} p d z$ на гранище - чисто мнимая величина. Следовательно, этот дифференциал аналитически продолжается на обратную сторону дубля как $-\partial_{\bar{z}} p d \bar{z}$. Наоборот, если задан симметричный абелев дифференциал на $\Sigma$, мы поместим насосы в его полюсах на лицевой стороне. Мощности насосов определяются вычетами в полюсах.

Отметим также, что любой симметричный абелев дифференциал $d \Omega$ на $\Sigma$ задает векторное поле на пространстве контуров. Именно, следует положить нормальную скорость точек контура равной

$$
V_{n}=-\frac{1}{2} \partial_{n} \operatorname{Re} \int^{z} d \Omega
$$

Если полюсы $d \Omega$ на $z$-плоскости фиксированы, $V_{n}$ есть векторное поле для некоторого лапласовского потока.

Например, пусть нефоть откачивается из точки $a \in \mathrm{D}_{\text {oil }}$ с единичной интенсивностью. Тогда

$$
p(z)=2 G(a, z)
$$

где $G$ - функция Грина задачи Дирихле в области $\mathrm{D}_{\text {оil }}$. Функция Грина определяется как такое решение уравнения Лапласа в $\mathrm{D}_{\text {оil }}$ с логарифмической особенностью вида 
$G(a, z) \rightarrow \ln |z-a|$ при $z \rightarrow a$, что $d G=0$ на границах. Следовательно, дифференциал $d W^{(a)}(z)=\partial_{z} p d z$ имеет простой полюс при $z=a$ с вычетом, равным 1 , и не имеет других особенностей в $\mathrm{D}_{\text {oil }}$. Аналитическое продолжение этого дифференциала на обратную сторону есть $-\overline{d W^{(a)}(z)}$, что дает простой полюс в зеркально-симметричной точке $a^{*}$ с вычетом, равным -1 . Построенный дифференциал является симметричным абелевым дифференциалом третьего рода (дипольным дифференциалом):

$$
d W^{(a)}= \begin{cases}2 \partial_{z} G(a, z) d z & \text { на лищевой стороне } \\ -2 \partial_{\bar{z}} G(a, z) d \bar{z} & \text { на обратной стороне. }\end{cases}
$$

ЗАмЕчАнИЕ 1 . При $g \geqslant 1$ эти условия еше не определяют дифференциал однозначно. Для однозначного определения нужна некоторая нормировка. Нормируем дифференциал $d W^{(a)}$ условием, что его а-периоды равны нулю. В зависимости от выбора циклов это означает наложение некоторых физических условий, например задание интенсивностей закачки или давлений в водяных каплях (подробнее см. [9]).

2.4. Иерархия Уизема. Структура иерархии Уизема проявляется в эволюции абелевых дифференциалов под действием лапласовских потоков $\Sigma \rightarrow \Sigma(T)$.

Голоморфная координата $z$ на лищевой стороне $\Sigma$ по построению не зависит от времени. Она задает некоторую связность в пространстве вешественных римановых поверхностей рода $g$. Соответственно абелевы дифференциалы приобретают зависимость от времени: $d W=d W(z, T)$. При любом фиксированном $z$ дифференциал $d W(z)$ есть некоторшй функционал в пространстве контуров, так что можно брать от него производные вдоль лапласовских векторных полей.

В работе [9] было показано, что лапласовский рост области $\mathrm{D}_{\text {oil }}$ (с нулевым поверхностным натяжением) эквивалентен системе уравнений Уизема для симметричных абелевых дифференциалов третьего рода $d W^{(a)}$ на дубле Шоттки области $\mathrm{D}_{\text {oil }}$. Основным результатом является следуюшая теорема [9].

Теорема 2. Для любой пары насосов (в точках $a, b \in \mathrm{D}_{\text {оil }}$ ) выполняется следующее соотношение:

$$
\partial_{T^{(a)}} d W^{(b)}=\partial_{T^{(b)}} d W^{(a)} .
$$

Это утверждение вытекает из (4) и вариационной формулы Адамара [14] для функции Грина $G(z, a)$. Из формулы Адамара следует, что величина $\partial_{T^{(a)}} G(b, c)$ симметрична относительно всех перестановок точек $a, b, c$.

Теорема 2 дает возможность отождествить лапласовские потоки с уиземовскими потоками на (расширенном) пространстве модулей вешественных римановых поверхностей. Система уравнений (5) для всех $a, b \in \mathrm{D}_{\text {oil }}$ составляет универсальную иерархию Уизема [11]. В теории солитонов эти уравнения получаются путем усреднения решений солитонных уравнений по быстрым осцилляциям [15]. 
Поскольку лапласовские векторные поля коммутируют, можно искать общее решение уравнения (5) в виде

$$
d W^{(a)}=-\partial_{T^{(a)}} d \Omega,
$$

где $d \Omega$ называется производящим дифференииалом.

ЗАмЕчАниЕ 2. Вообще говоря, производящий дифференциал может иметь особенности очень сложной природы. Из уравнений (6), однако, следует, что все эти особенности остаются неподвижными под действием лапласовских потоков. Другими словами, они являются интегралами движения. Единственный параметр, который меняется в процессе лапласовского роста со стоком в точке $a,-$ это вычет дифференциала $d \Omega$ в полюсе в точке $a$. Это, в частности, означает, что лапласовские потоки с точечными насосами могут добавлять к особенностям дифференциала $d \Omega$ лишь простые полюсы.

В следующем разделе мы рассмотрим важный специальный случай, когда все особенности производящего дифференциала являются полюсами, т.е. $d \Omega$ мероморфен на $\Sigma$.

\section{3. АЛГЕБРАИЧЕСКИЕ ОБЛАСТИ}

Семейства специальных решений уравнений Уизема с мероморфным производяшим дифференциалом были построены в [10]. В [9] было показано, что они описывают лапласовский рост алгебраических областей. Ясно, что алгебраическая область остается алгебраической в процессе лапласовского роста с точечными насосами. Этот факт позволяет редуцировать бесконечномерное конфигурационное пространство контуров к конечномерному.

3.1. Функция Шварца. Для более явного описания класса алгебраических областей, с которыми мы собираемся иметь дело, рассмотрим аналитическое продолжение функции $z$, определенной на $\mathrm{D}_{\text {oil }}$, на обратную сторону дубля Шоттки.

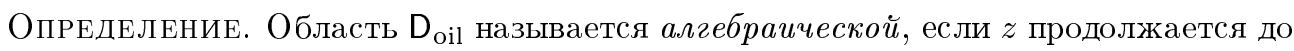
мероморфной функции на ее дубле Шоттки.

Чтобы прояснить смысл этого определения, напомним понятие функции Шварца [16]. Пусть дана некоторая область с границей на плоскости; ее функция Шварца - это аналитическое продолжение функции $\bar{z}$ с границы. Отметим, что для того чтобы такое аналитическое продолжение сушествовало, гранища должна быть аналитической, а не просто гладкой. По определению функция Шварца $S(z)$ - это функция, аналитическая в некоторой окрестности граничного контура (контуров) такая, что $S(z)=\bar{z}$ на контуре. Если она существует, функция Шварца должна удовлетворять "условию унитарности" $S(\overline{S(z)})=\bar{z}$, которое означает, что функция $\overline{S(\bar{z})}$ обратна к $S(z)$. Вообще говоря, аналитические продолжения с разных компонент гранищы многосвязной области могут давать разные функции. Алгебраические области соответствуют весьма специальной ситуации, когда эти аналитические продолжения дают одну и ту же функцию, определенную везде в $\mathrm{D}_{\mathrm{oil}}$. 
Если функция Шварца сушествует, $\overline{S(z)}$ представляет собой аналитическое продолжение функции $z$ на обратную сторону дубля. Следовательно, плоская область является алгебраической тогда и только тогда, когда функция Шварца продолжается до мероморфной функции в этой области.
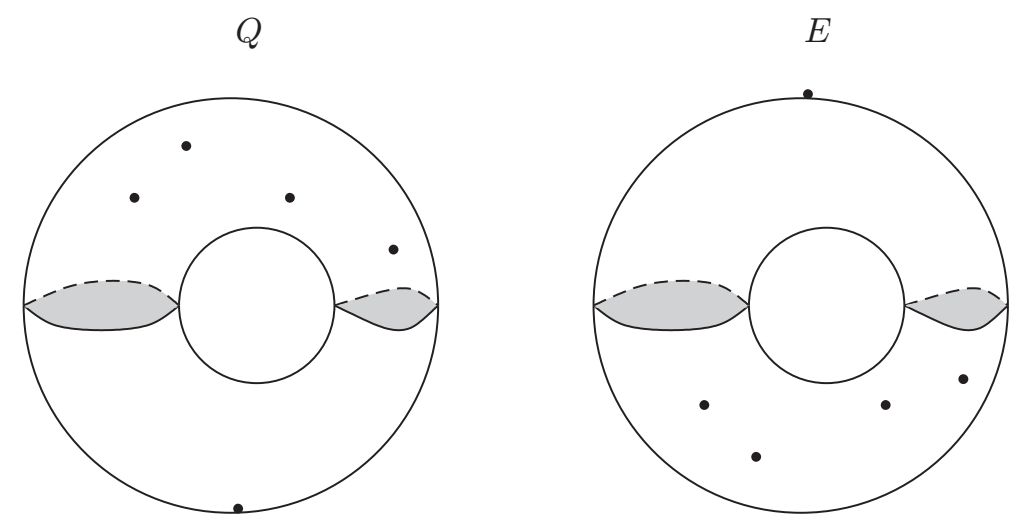

Рис. 2. Полюсы функций $Q$ и $E$ на дубле Шоттки $(g=1)$. Верхняя и нижняя половины тора схематически изображают соответственно лицевую и обратную стороны дубля.

Обозначим через $P \in \Sigma$ произвольную точку дубля Шоттки алгебраической области. Рассмотрим следуюшую функцию на $\Sigma$ :

$$
Q(P)= \begin{cases}S(z), & \text { если } P \text { принадлежит лицевой стороне, } \\ \bar{z}, & \text { если } P \text { принадлежит обратной стороне }\end{cases}
$$

где $z=z(P)$ - это $z$-координата точки $P=(z, \sigma)$ на $\Sigma$. Согласно определению функции Шварца $Q(P)$ непрерьвна при переходе через контур $\gamma$, и, значит, она корректно определена как мероморфная функция на $\Sigma$. Наряду с $Q(P)$ введем “зеркально-симметричную” функцию

$$
E(P)= \begin{cases}z, & \text { если } P \text { принадлежит лицевой стороне, } \\ \overline{S(z)}, & \text { если } P \text { принадлежит обратной стороне, }\end{cases}
$$

которая также мероморфна на $\Sigma$. Она доставляет упомянутое выше мероморфное продолжение функции $z$ на весь дубль. Отметим, что функции $Q$ и $E$ связаны соотношением

$$
S(E(P))=Q(P)
$$

и переходят друг в друга (с комплексным сопряжением) при антиголоморфной инволюции:

$$
Q\left(P^{*}\right)=\overline{E(P)}
$$

Пусть функция Шварца в $\mathrm{D}_{\text {оil }}$ имеет полюсы в некоторых точках $p_{j} \in \mathrm{D}_{\text {оil }}$ с кратностями $n_{j}$. Здесь и далее предполагается, что точки $p_{j}$ находятся в обшем положении. 
Положим $N=\sum_{j} n_{j}$. Тогда число капель не может превьшать $N+1$, или, что то же самое, род дубля Шоттки $g \leqslant N$. Эта грубая оценка ${ }^{2)}$ следует из того факта, что любая нетривиальная функция на римановой поверхности рода $g$ имеет по крайней мере $g+1$ полюсов, посчитанных с учетом их кратности (если все данные находятся в общем положении). Оценка для числа капель получается применением этого утверждения $\mathrm{k}$ функции $Q$.

В итоге мы заключаем, что на дубле Шоттки алгебраической области сушествуют две мероморфные функции, связанные инволюцией (8). Обозначения $Q, E$ для них выбраны для того, чтобы подчеркнуть связь с подходом, развитым в [11].

ЗАмЕчАниЕ 3. Класс алгебраических областей, введенных здесь и в [4], совпадает с классом "квадратурных областей" из [17]. Класс областей, соответствуюших "алгебраическим орбитам" уравнений Уизема [11], более широк. Он включает области, для которых порождаюший дифференшиал на $\Sigma$ может иметь "скачки" на определенных циклах, а в остальном мероморфен.

3.2. Комплексная кривая, связанная с алгебраической областью. Функция Шварца алгебраических областей является алгебраической функиией, т.е. удовлетворяет некоторому полиномиальному уравнению $R(S(z), z)=0$. Простейшее доказательство этого утверждения можно получить, рассмотрев аналитические продолжения функций $z, S(z)$ на весь дубль, т.е. функций $E, Q$.

Пусть $S(z)$ имеет $N$ полюсов в $\mathrm{D}_{\text {оil }}$ (с учетом кратностей), тогда функция $Q$ имеет $N+1$ полюсов на $\Sigma$ ( $N$ полюсов на лицевой стороне и простой полюс в бесконечности на обратной стороне). Функция $E$ тоже имеет $N+1$ аналогичных полюсов. Следовательно, мы имеем две мероморфные функции с $N+1$ полюсами на гладкой римановой поверхности. Простая теорема алгебраической геометрии утверждает, что эти функции связаны полиномиальным уравнением степени $N+1$ по каждой переменной:

$$
R(Q, E)=\sum_{n, m=0}^{N+1} A_{n m} Q^{n} E^{m}=0 .
$$

Из симметрии относительно инволюции следует, что матрица коэффициентов эрмитова: $A_{n m}=\overline{A_{m n}}$. Рассмотрев соотношение между $Q$ и $E$ на лицевой стороне дубля, мы получаем полиномиальное уравнение $R(S(z), z)=0$. Условие унитарности для функции Шварца эквивалентно эрмитовости коэффициентов этого полинома.

Уравнение $R(\tilde{z}, z)=0$ определяет комплексную алгебраическую кривую $\Gamma$, которая может служить еше одной реализацией римановой поверхности $\Sigma$. Граничньй контур восстанавливается как множество вешественных решений $x, y$ уравнения $R(x-i y$, $x+i y)=0$. Это вешественное сечение кривой $\Gamma$.

\footnotetext{
2) Эта оценка действительно довольно грубая. Ее можно улучшить, приняв во внимание условия вешественности. Например, в случае $N=1$ наше неравенство дает $g \leqslant 1$, в то время как более тщательный анализ (см., например, теорему 7 и следствие 7.1 в [17]) показывает, что $g=0$.
} 
ЗАмЕчАниЕ 4. Мы видим, что граница алгебраической области всегда описывается полиномиальным уравнением. Обратное неверно. Произвольно взятое полиномиальное уравнение с эрмитовыми коэффициентами, вообше говоря, не задает алгебраическую область.

Несмотря на то что $\Sigma$ - гладкая поверхность, кривая $\Gamma$ не гладкая при $N>0$. На ней имеются особые точки (двойные точки в случае общего положения). Это можно увидеть, сравнив род дубля с верхней оценкой для рода кривой $\Gamma$. Для гладкой кривой вида (9) род равен $N^{2}$, поэтому тот факт, что род, на самом деле, не превьшает $N$, как раз и означает наличие особых точек на кривой $\Gamma$.

Как возникают особые точки? Рассмотрим отображение $P \mapsto(Q(P), E(P))$ из $\Sigma$ в $\Gamma$, переводящее точку $P$ в пару комплексных чисел $(Q(P), E(P))$, связанных полиномиальным уравнением. Если две различные точки $\Sigma$ отображаются в одну точку на $\Gamma$ (т.е. значения обеих функций $Q, E$ для них одинаковы), то эта точка, очевидно, является особой точкой на кривой, а именно точкой самопересечения (двойной точкой). Эта ситуация показана на рис. 3. Говоря коротко, отображение дубля Шоттки на кривую склеивает точки. Это значит, что $\Sigma$, вообще говоря, нельзя реализовать как гладкую алгебраическую кривую в $\mathbb{C}^{2}$.

Дадим более точное описание точек, которые могут быть склеены. Две различные точки $P_{1}, P_{2} \in \Sigma$ склеиваются, если $Q\left(P_{1}\right)=Q\left(P_{2}\right)$ и $E\left(P_{1}\right)=E\left(P_{2}\right)$. Как легко видеть, это возможно, только если $P_{1}$ и $P_{2}$ находятся на разных сторонах дубля. Положим $P_{1}=\left(z_{1},+\right), P_{2}=\left(z_{2},-\right), z_{1}, z_{2} \in \mathrm{D}_{\text {оil }}$. Тогда эти точки склеиваются, если $S\left(z_{1}\right)=\bar{z}_{2}$ и $S\left(z_{2}\right)=\bar{z}_{1}$. Может показаться, что второе равенство следует из первого в силу условия унитарности. Однако условие унитарности имеет место для полной алгебраической функции $S(z)$, тогда как $S(z)$ в написанных вьшше равенствах означает одну и ту же ветвь функции Шварца, полученную аналитическим продолжением $\bar{z}$ в $\mathrm{D}_{\text {oil }}$. Тем самым, для определения точек $z_{1}$ и $z_{2}$ мы имеем два независимых условия. Другими словами, $z_{1}$ (или $z_{2}$ ) - это точка в $\mathrm{D}_{\text {оil }}$, в которой две или больше ветви функции Шварца принимают одинаковые значения.

Тем не менее (гладкий) дубль Шоттки всегда можно реализовать как гладкую комплексную кривую $\widetilde{\Gamma}$ в пространстве большего числа измерений. Эта кривая получается из $\Gamma$ в результате применения процедуры десингуляризации. В случае точки самопересечения это просто означает рассмотрение третьей мероморфной функции на $\Sigma$ (скажем, $K(P))$ такой, что она принимает разные значения в точках, которые склеиваются отображением $P \mapsto(Q(P), E(P))$ (см. рис. 3). Тогда кривая задается системой полиномиальных уравнений $R_{1}(Q, K)=0, R_{2}(K, E)=0$ в $\mathbb{C}^{3}$. Если эта кривая все еше особая, надо повторить процедуру, пока не получится гладкая кривая $\widetilde{\Gamma}$.

Полученная таким образом кривая $\widetilde{\Gamma}$ изоморфна римановой поверхности функции Шварца. Последнюю можно реализовать как разветвленное накрытие $z$-плоскости. В этой реализации антиголоморфная инволюция совпадает с отражением Шварца $z \mapsto$ $\overline{S(z)}$, которое переводит точку $z$ в ее "зеркальное отражение" относительно граничного 


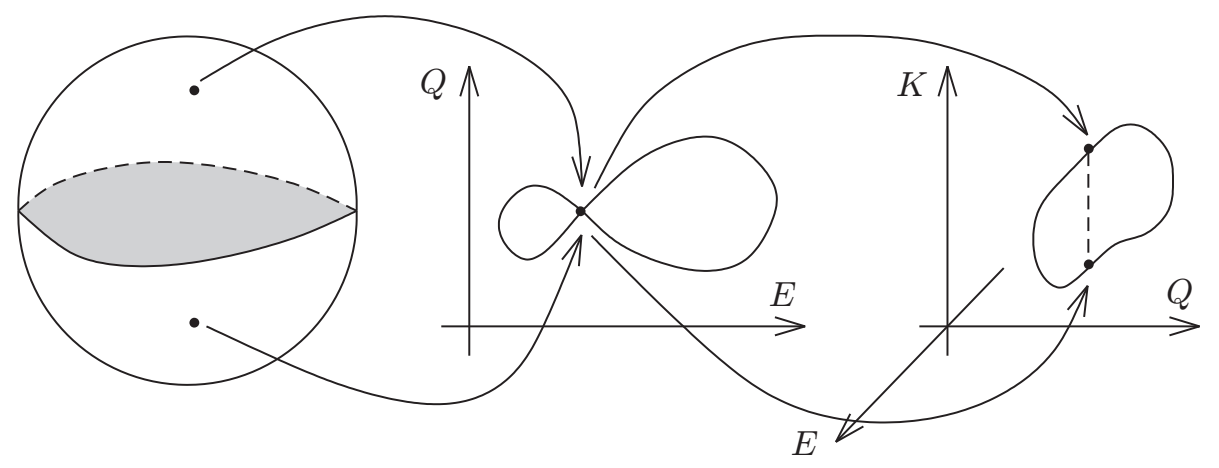

Рис. 3. От дубля Шоттки к особой кривой $\Gamma$ и затем к гладкой кривой $\widetilde{\Gamma}$.

контура ${ }^{3)}$.

3.3. Производящий дифференциал. Производящий дифференциал для алгебраической области имеет следуюший явньй вид:

$$
d \Omega=Q d E= \begin{cases}S(z) d z & \text { на лицевой стороне } \\ \bar{z} d \overline{S(z)} & \text { на обратной стороне. }\end{cases}
$$

Чтобы это доказать, надо проверить справедливость равенств (6) для всех векторных полей $\partial_{T^{(a)}}$. Это равенство немедленно следует из поведения особенностей функции Шварца под действием лапласовских потоков.

ПРЕДЛОЖЕНИЕ. Положение полюсов функиии Шварца любой алгебраической области остается неизменным под действием всех лапласовских потоков с точечныцми насосами. Вычеты в полюсах тоже не меняются, за исключением вычета в точке, в которой находится источник:

$$
\left.\partial_{T^{(b)} \operatorname{res}}\right|_{z=a}(S(z) d z)= \begin{cases}0, & \text { если } b \neq a \\ -1, & \text { если } b=a .\end{cases}
$$

ЗАМЕчАниЕ 5. Если функция $S(z)$ в начальный момент была регулярна в точке $a$, лапласовский поток со стоком в $a$ приводит к образованию простого полюса с вычетом, равным $-T^{(a)}$, в этой точке.

\footnotetext{
${ }^{3)}$ Такая геометрическая интерпретация требует некоторой осторожности. Если точка $z$ находится недалеко от контура, так что ее образ лежит на том же самом листе накрытия, это действительно выглядит, как отражение в обычном смысле. Однако, если точка $z$ лежит вдали от контура, ее образ, проходя через разрез, попадает на другой лист. В этом случае условие унитарности (инволютивность отражкения) выполняется только при выборе подходящей ветви функции Шварца.
}

2 Теоретическая и математическая физика, т. 142, № 2, 2005 г. 
Один из методов доказательства этого предложения заключается в рассмотрении функции

$$
C(z)=\oint_{\gamma} \frac{\bar{\xi} d \xi}{z-\xi},
$$

вариация которой при лапласовских потоках может быть найдена прямым вычислением. С другой стороны, как это следует из свойств интеграла Коши, особенности функции Шварца вне $\gamma$ (т.е. в $\mathrm{D}_{\text {oil }}$ ) совпадают с особенностями аналитического продолжения $C(z)$ из внутренности во внешность контура $\gamma$.

Из этого предложения следует, что единственная особенность дифференциала $\partial_{T^{(a)}}(Q d E)$ на лищевой стороне - это простой полюс в точке $a$ с вычетом, равным -1 . Условие унитарности означает, что этот дифференциал является чисто мнимым на $\gamma$. В самом деле, действуя на условие унитарности $S(\overline{S(z, T)}, T)=\bar{z}$ операторами $\partial_{\bar{z}}$ и $\partial_{T}$, получим тождества

$$
\begin{gathered}
S^{\prime}(\overline{S(z)}) \overline{S^{\prime}(z)}=1, \\
\partial_{T} S(\overline{S(z)}, T)+S^{\prime} \overline{(\overline{S(z)})} \partial_{T} \overline{S(z, T)}=0,
\end{gathered}
$$

где $\partial_{T}$ означает любое из $\partial_{T^{(a)}}$. Объединяя их, находим

$$
\partial_{T} \overline{S(z, T) d z}=-\partial_{T} S(\overline{S(z)}, T) d \overline{S(z)}
$$

что дает $\overline{\partial_{T} S d z}=-\partial_{T} S d z$ на $\gamma$. Приняв во внимание условие нормировки, мы заключаем, что

$$
\partial_{T^{(a)}}(Q d E)=-d W^{(a)} \quad \text { для всех } a \in \mathrm{D}_{\text {oil }} \text {. }
$$

Зафиксируем точку а и положим $T=T^{(a)}, W=W^{(a)}$. Вместо функции $z$ в качестве связности (в смысле [12]) на пространстве вешественных римановых поверхностей выберем функцию $W=W(z, T)$, т.е. потребуем постоянства $W$ при действии векторным полем $\partial_{T}$. Уравнение (13) примет при этом вид

$$
\partial_{T} E(W, T) d Q-\partial_{T} Q(W, T) d E=d W
$$

В односвязном случае оно превращается (после ограничения на $\gamma$ и отождествления $E=f, Q=\bar{f}, i W=\phi)$ в уравнение лапласовского роста (2).

\section{4. УРАВНЕНИЯ УИЗЕМА ДЛЯ ЛАПЛАСОВСКОГО РОСТА АЛГЕБРАИЧЕСКИХ ОБЛАСТЕЙ}

В этом разделе иерархия Уизема (5) будет представлена как конечная система дифференциальных уравнений для точек ветвления функции Шварца и дан общий вид решения этой системы обобшенным методом годографа. 
4.1. Точки ветвления функции Шварца. Пусть дана некоторая алгебраическая область; рассмотрим нули дифференциала $d E$ на ее дубле Шоттки. Поскольку на лицевой стороне $E=z$, все эти нули лежат на обратной стороне. Обозначим их $\lambda_{k}^{*}=$ $\left(\lambda_{k},-\right)$, так что $\lambda_{k}-$ их проекции на $z$-плоскость. Поскольку $0=d E\left(\lambda_{k}^{*}\right)=d \overline{S\left(\lambda_{k}\right)}$, точки $\lambda_{k}$ являются нулями производной функции Шварца в $\mathrm{D}_{\mathrm{oil}}: S^{\prime}\left(\lambda_{k}\right)=0, \lambda_{k} \in \mathrm{D}_{\text {oil }}$.

Если даны полюсы функции Шварца и число капель, количество таких точек может быть найдено из следующих соображений. Дифференциал $d E$ имеет полюсы порядка $n_{j}+1$ в точках $\left(p_{j},-\right)$ на обратной стороне дубля (напомним, что $p_{j}-$ полюсы функции Шварца) и полюс второго порядка в бесконечности на лищевой стороне. Общее число полюсов равно $2+\sum_{j}\left(n_{j}+1\right)$ с учетом кратности. Для любого мероморфного дифференциала на гладкой римановой поверхности рода $g$ разность между числом его нулей и полюсов равна $2 g-2$. Следовательно, число нулей дифференциала $d E$ есть

$$
M=2 g+\sum_{j}\left(n_{j}+1\right) .
$$

В частности, если все полюсы $p_{j}$ функции Шварца простые $\left(n_{j}=1\right)$, то

$$
M=2(g+N) .
$$

В ситуации общего положения все нули дифференциала $d E$ простые. В этом предположении критические значения функции $E$, т.е. значения $E$ в нулях $d E$,

$$
E_{k}:=E\left(\lambda_{k}^{*}\right)=\overline{S\left(\lambda_{k}\right)}
$$

играют роль локальных координат в пространстве алгебраических областей с данными $N$ и $g$ (см. [10]). Они являются образами точек $\lambda_{k}$ при отражении Шварца. Их геометрический смысл становится ясным из разложения условия унитарности вблизи точек $\lambda_{k}\left(\right.$ или $\left.E_{k}\right)$. В результате имеем

$$
S(z)=S\left(E_{k}\right)+\alpha_{k} \sqrt{z-E_{k}}+\cdots, \quad z \rightarrow E_{k} .
$$

Следовательно, $E_{k}$ - точки ветвления функции Шварца. В ситуации общего положения их порядок равен двум. Если все полюсы функции Шварца простые, все $2(g+N)$ точек ветвления (см. (16)) находятся внутри капель воды. В этом случае однозначную ветвь функции Шварца можно задать, сделав $g+N$ разрезов между ними.

4.2. Динамика точек ветвления. Здесь мы выведем дифференциальные уравнения для динамики точек ветвления функции Шварца $E_{i}=E_{i}(T)$.

Разложим равенство $(5)$ на $\Sigma$ в ряд в тех точках, где $E$ не является хорошим локальным параметром, т.е. в нулях $\lambda_{k}^{*}$ дифференциала $d E$. Вблизи этих точек локальными параметрами служат $\sqrt{E-E_{k}}$, так что разложения имеют вид

$$
\begin{aligned}
& W^{(a)}=W^{(a)}\left(\lambda_{k}^{*}\right)+C_{k}^{(a)} \sqrt{E-E_{k}}+O\left(E-E_{k}\right), \\
& \partial_{T^{(b)}} d W^{(a)}=\frac{C_{k}^{(a)} \partial_{T^{(b)}} E_{k} d E}{2\left(E-E_{k}\right)^{3 / 2}}+O\left(\frac{d E}{\sqrt{E-E_{k}}}\right) .
\end{aligned}
$$


Вид разложения для $W^{(b)}$ аналогичен. Сравнивая сингулярные части вблизи точек ветвления, для всех $k$ получаем равенства

$$
C_{k}^{(b)} \partial_{T^{(a)}} E_{k}=C_{k}^{(a)} \partial_{T^{(b)}} E_{k}, \quad C_{k}^{(b)} d W^{(a)}\left(\lambda_{k}^{*}\right)=C_{k}^{(a)} d W^{(b)}\left(\lambda_{k}^{*}\right)
$$

Разделив первое равенство на второе, приходим к замкнутой системе дифференциальных уравнений гидродинамического типа на точки ветвления $E_{k}$

$$
\partial_{T^{(a)}} E_{k}=V_{k}^{(a b)}\left(E_{1}, \ldots, E_{M}\right) \partial_{T^{(b)}} E_{k}, \quad k=1, \ldots, M
$$

где "групповые скорости" $V_{k}^{(a b)}$ даются формулой

$$
V_{k}^{(a b)}=\left.\left(\frac{d W^{(a)}(z)}{d W^{(b)}(z)}\right)\right|_{z=E_{k}} .
$$

В общем случае $V_{k}^{(a b)}$ - это сложные нелинейные функции от $E_{k}$. Система (19) диагональна по отношению к переменным $E_{k}$, так что точки ветвления функции Шварца являются инвариантами Римана.

Допустимые данные Коши для системы (19) даются решениями системы обыкновенных дифференциальных уравнений вида

$$
\partial_{T^{(a)}} E_{k}=\left.\left(\frac{d W^{(a)}(z)}{d S(z)}\right)\right|_{z=E_{k}}
$$

которая эквивалентна “струнному уравнению” (14). Аналогично вычислению, проделанному выше, эти уравнения получаются разложением уравнения (14) вблизи нулей $d E$. Отметим, что $\partial_{T} E(W, T)=\partial_{T} E_{k}$ в нулях $\lambda_{k}^{*}$ дифференциала $d E$.

Решения этих уравнений строятся с помощью обобшенного метода годографа [12], [10]. В версии работы [10] эти решения получаются путем представления производящего дифференциала в виде

$$
Q d E=d \Lambda-T^{(a)} d W^{(a)}-T^{(b)} d W^{(b)},
$$

где $d \Lambda$ - некоторый дифференциал с неподвижными (не зависящими от $T$ ) особенностями, $\partial_{T}(\Delta \Lambda(z))=0$ ( $\Delta$ - оператор Лапласа). В случае алгебраических областей $d \Lambda$ - мероморфный дифференциал с фиксированными главными частями во всех полюсах. Поскольку функция $Q$ регулярна на обратной стороне $\Sigma$ (кроме полюса в бесконечности), правая часть (22) должна обращаться в нуль в точках $\lambda_{k}^{*}$. Это условие приводит к "соотношениям годограф̆а"

$$
T^{(a)} d W^{(a)}\left(E_{k}\right)+T^{(b)} d W^{(b)}\left(E_{k}\right)=d \Lambda\left(E_{k}\right),
$$


или, в эквивалентной форме, к

$$
T^{(b)}+V_{k}^{(a b)} T^{(a)}=U_{k}^{(b)}
$$

для всех $k=1, \ldots, M$, где

$$
U_{k}^{(b)}=\left.\left(\frac{d \Lambda(z)}{d W^{(b)}(z)}\right)\right|_{z=E_{k}} .
$$

Эти равенства дают неявные решения уравнений Уизема (19) и "струнного уравнения" (21). Лапласовский рост со стоком в точке $b$ соответствует изменению $T^{(b)}$ при фиксированном $T^{(a)}$.

Как правило, построенные решения имеют точки бифуркации. В приложениях к нелинейным волнам такая точка означает, что фронт волны опрокидывается. В лапласовском росте точки бифуркации соответствуют нефизическим сингулярностям (каспам) движущейся гранищы раздела, которые, однако, являются типичными [1], [6] в пределе нулевого поверхностного натяжения.

\section{5. ПРИМЕР: $N=1$}

Рассмотрим пример, в котором функция Шварца имеет простой полюс в точке $p \in$ $\mathrm{D}_{\text {oil }}$ и регулярна в остальных точках $\mathrm{D}_{\text {oil }}(N=1)$. Наше изложение следует работе [18]. Вблизи полюса имеем

$$
S(z)=\frac{\mu}{p-z}+O(1), \quad z \rightarrow p, \quad p \in \mathrm{D}_{\mathrm{oil}}
$$

где вычет положен равным $-\mu$. В соответствии с общей теорией функция $S(z)$ при $N=1$ удовлетворяет алгебраическому уравнению

$$
\sum_{l, k=0}^{2} A_{l k} S^{l}(z) z^{k}=0
$$

квадратичному по каждой из переменных. Следовательно, риманова поверхность функции Шварца имеет два листа над z-плоскостью. Один из них является физическим. Это тот лист, где выполняется соотношение $S(z)=\bar{z}$ на контуре. В дальнейшем мы будем называть его первым (или верхним) листом. Полюс $p$ лежит на этом листе. Аналитическое продолжение функции Шварца внутрь водяных капель имеет еше один полюс в точке, которая дается значением функции $E$ в простом полюсе функции $Q$ в бесконечности на обратной стороне дубля Шоттки. Обозначим этот полюс через $q$. Он лежит на втором (нефизическом) листе. В итоге особенности функции Шварца следуюшие:

$$
S(z)= \begin{cases}\frac{\mu}{p-z}+O(1), & z \rightarrow p \text { на первом (физическом) листе } \\ \frac{\nu}{q-z}+O(1), & z \rightarrow q \text { на втором листе. }\end{cases}
$$


Из условия унитарности следует, что

$$
S(z)= \begin{cases}\bar{q}-\frac{\bar{\nu}}{z}+O\left(z^{-2}\right), & z \rightarrow \infty \text { на первом листе } \\ \bar{p}-\frac{\bar{\mu}}{z}+O\left(z^{-2}\right), & z \rightarrow \infty \text { на втором листе. }\end{cases}
$$

Отсюда находим все особенности дифференциала $S d z$ на римановой поверхности функции Шварца. Это два простых полюса в точках $p$ и $q$ на разных листах (с вычетами $-\mu$, $-\nu)$ и два полюса второго порядка в двух бесконечно удаленных точках (с вычетами $\bar{\nu}$ и $\bar{\mu})$. Сумма вычетов должна равняться нулю. Отсюда следует, что $\mu+\nu-$ вешественное число:

$$
\mu+\nu=\bar{\mu}+\bar{\nu} .
$$

Подставив главные части разложения функции Шварца (24) в уравнение

$$
\tilde{z}^{2} z^{2}+b \tilde{z}^{2} z+\bar{b} \tilde{z} z^{2}+c \tilde{z} z+d \tilde{z}^{2}+\bar{d} z^{2}+e \tilde{z}+\bar{e} z+h=0
$$

где $\tilde{z}=S(z)$, можно фиксировать все неизвестные коэффициенты, кроме свободного члена $h($ cp. с [19]):

$$
\begin{array}{ll}
b=-p-q, & c=|p+q|^{2}+\mu+\nu, \\
d=p q, & e=-p q(\bar{p}+\bar{q})-p \nu-q \mu
\end{array}
$$

(предполагается, что $\mu, \nu \neq 0$ ).

Сколько капель может существовать при $N=1$ ? В общем случае уравнение вида (27) задает гладкую кривую рода 1 (эллиптическую кривую). Вешественное сечение этой кривой (множество точек таких, что $\tilde{z}=\bar{z}$ ) может состоять не более чем из двух несвязных замкнутых контуров на плоскости. В случае двух контуров более детальньй анализ показывает (см., например, доказательство теоремы 7 в [17] или соответствующий пример в [18]), что один из них обязательно лежит на нефизическом листе. Следовательно, две капли при $N=1$ невозможны. Другими словами, все алгебраические области с $N=1$ односвязны. В этом случае кривая (27) - это рациональная (рода 0) кривая с особыми точками (гладкая кривая рода 1 вида (27) не может быть бирационально эквивалентна дублю Шоттки односвязной области, имеюшему род 0). При данных коэффициентах $b, c, d, e$ это условие определяет коэффициент $h$.

Решив квадратное уравнение (27), можно найти функцию Шварца в более явном виде:

$$
S(z)=\frac{1}{2}(\bar{p}+\bar{q})+\frac{p \nu+q \mu-(\mu+\nu) z+(\bar{q}-\bar{p}) \sqrt{P_{4}(z)}}{2(z-p)(z-q)} .
$$

Здесь $P_{4}(z)$ - полином четвертой степени со старшим членом $z^{4}$. Вообще говоря, функция $\sqrt{P_{4}(z)}$ имеет четыре точки ветвления, которые являются простыми корнями полинома $P_{4}$. Кривая вырождается, когда два корня (скажем, $E_{3}$ и $\left.E_{4}\right)$ сливаются друг с другом:

$$
\sqrt{P_{4}(z)}=\left(z-E_{3}\right) \sqrt{P_{2}(z)}, \quad P_{2}(z)=\left(z-E_{1}\right)\left(z-E_{2}\right) .
$$


При этом остается только один разрез между $E_{1}, E_{2}$, и кривая имеет род 0 (рис. 4 ). Второй разрез вырождается в двойную точку кривой. Легко видеть, что первый (физический) лист соответствует той ветви функции $\sqrt{P_{2}(z)}$, для которой $\sqrt{P_{2}(z)} \rightarrow z$ при $z \rightarrow \infty$.

Концы разреза $E_{1}, E_{2}$, вообще говоря, не могут быть выражены в явном виде простыми формулами (в данном случае надо решать уравнение четвертой степени). Мы знаем, однако, что они удовлетворяют уравнениям гидродинамического типа, решения которых можно представить в форме соотношений годографа (22).

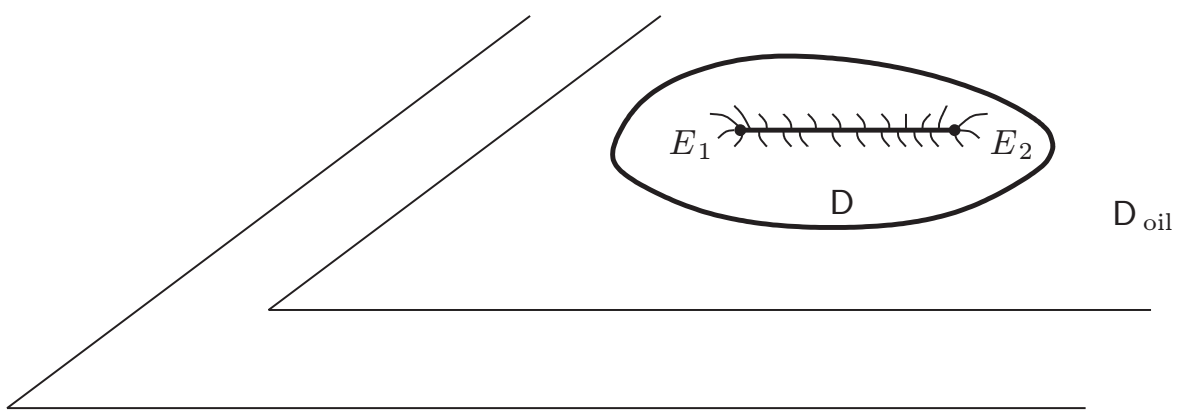

Рис. 4. Риманова поверхность функции Шварца при $N=1$ двулистно накрывает $z$-плоскость. Отображение $E: \Sigma \rightarrow \mathbf{P}=\mathbb{C} \cup\{\infty\}$ является двулистным накрытием, т.е. каждая точка $z \in \mathbf{P}$ имеет два прообраза (с учетом кратности). Образ лицевой стороны дубля это область $\mathrm{D}_{\text {oil }}$ на первом листе. Образ обратной стороны дубля - остальная часть двулистной римановой поверхности, т.е. внутренность граничного контура на первом листе (область D) и весь второй лист.

Соотношения годографа следуют из аналитических свойств дифференциала $S d z$. Это мероморфный дифференциал на двулистной римановой поверхности с разрезом между $E_{1}, E_{2}$. Он имеет два простых полюса и два полюса второго порядка. Простые полюсы находятся в точке $p$ на первом листе и в точке $q$ на втором листе с вычетами $-\mu,-\nu$, соответственно. Полюсы второго порядка - это $\infty^{(1)}\left(\infty\right.$ на первом листе) и $\infty^{(2)}(\infty$ на втором листе) с вычетами $\bar{\nu}$ и $\bar{\mu}$, соответственно. Следовательно, мы можем написать

$$
S d z=\bar{q} d W_{+}+\bar{p} d W_{-}-\mu d W_{p^{(1)}, \infty^{(1)}}+\bar{\mu} d W_{\infty^{(2)}, q^{(2)}}-(\bar{\mu}-\nu) d W_{\infty}^{(1)}, q^{(2)} .
$$

Здесь $d W_{ \pm}-$мероморфные дифференциалы с единственным полюсом второго порядка в бесконечности на первом и втором листах, соответственно, и с главной частью $(1+$ $\left.O\left(z^{-2}\right)\right) d z$ при $z \rightarrow \infty ; d W_{a, b}-$ дипольньй мероморфньй дифференциал с простыми полюсами в $a$ и $b$ с вычетами \pm 1 , соответственно. Верхние индексы указывают лист, на котором лежат точки. Например, $d W_{\infty}^{(1)}, q^{(2)}$ означает дипольный дифференциал с полюсами в бесконечности на первом листе и в точке $q$ на втором листе. Явный вид этих 
дифференциалов таков:

$$
\begin{aligned}
d W_{ \pm} & =\left[\sqrt{P_{2}(z)} \pm\left(z-\frac{E_{1}+E_{2}}{2}\right)\right] \frac{d z}{2 \sqrt{P_{2}(z)}} \\
d W_{a, b} & =\left(\frac{\sqrt{P_{2}(z)}+\sqrt{P_{2}(a)}}{z-a}-\frac{\sqrt{P_{2}(z)}+\sqrt{P_{2}(b)}}{z-b}\right) \frac{d z}{2 \sqrt{P_{2}(z)}} .
\end{aligned}
$$

Подставляя явные выражения для дифференциалов в (29), находим $S(z)$ в виде

$$
S(z)=f_{1}(z)+\frac{f_{2}(z)}{\sqrt{P_{2}(z)}}
$$

где

$$
\begin{aligned}
& 2 f_{1}(z)=\bar{p}+\bar{q}+\frac{\mu}{p-z}+\frac{\nu}{q-z}, \\
& 2 f_{2}(z)=\frac{1}{2}(\bar{q}-\bar{p})\left(2 z-E_{1}-E_{2}\right)+\bar{\mu}-\bar{\nu}+\mu \frac{\sqrt{P_{2}(p)}}{z-p}+\nu \frac{\sqrt{P_{2}(q)}}{z-q} .
\end{aligned}
$$

Уравнения годографа следуют из того факта, что значения $S\left(E_{1}\right)$ и $S\left(E_{2}\right)$ конечны, т.е. $f_{2}\left(E_{1}\right)=f_{2}\left(E_{2}\right)=0$.

Если все параметры вешественны и $q<E_{1}, E_{2}<p$, система уравнений годографа принимает вид

$$
\begin{aligned}
& \mu\left(\sqrt{\frac{p-E_{2}}{p-E_{1}}}-\sqrt{\frac{E_{2}-q}{E_{1}-q}}\right)+T\left(\sqrt{\frac{E_{2}-q}{E_{1}-q}}-1\right)=\frac{1}{2}\left(E_{2}-E_{1}\right)(p-q), \\
& \mu\left(\sqrt{\frac{p-E_{1}}{p-E_{2}}}-\sqrt{\frac{E_{1}-q}{E_{2}-q}}\right)+T\left(\sqrt{\frac{E_{1}-q}{E_{2}-q}}-1\right)=-\frac{1}{2}\left(E_{2}-E_{1}\right)(p-q),
\end{aligned}
$$

где квадратные корни из положительных чисел считаются положительными. Вешественные параметры $\mu, T$, где

$$
T=\mu-\bar{\nu}=\frac{1}{2 \pi i} \oint_{\gamma} S(z) d z=\frac{\text { площаде }(\mathrm{D})}{\pi},
$$

являются локальными координатами в двумерном многообразии контуров, полученных из круга (радиуса $r_{0}$ ) в результате процессов лапласовского роста с нефтяными насосами в точках $p$ и $\infty$. В этой интерпретации $T-r_{0}^{2}$ равно полному объему откачанной нефти, а $\mu$ равно количеству нефти, откачанной из точки $p$.

Благодарности. Автор благодарен О. Агаму, Э. Беттельхайму, П. Вигману, И. Кричеверу, А. Маршакову, М. Минееву-Вайнштейну, Р. Теодореску за сотрудничество и полезные обсуждения. Эта работа была частично поддержана грантом РФФИ № 04-01-00642, грантом поддержки ведуших научных школ НШ-1999.2003.2 и проектом LDRD 20020006ER "Unstable Fluid/Fluid Interfaces" Лос-Аламосской Национальной лаборатории. 


\section{Список литературы}

[1] D. Bensimon, L. P. Kadanoff, S. Liang, B. I. Shraiman, C. Tang. Rev. Mod. Phys. 1986. V. 58. P. 977-999.

[2] S. Richardson. J. Fluid Mech. 1972. V. 56. P. 609-618; Eur. J. Appl. Math. 1994. V. 5. P. 97-122; Phil. Trans. R. Soc. London A. 1996. V. 354. P. 2513-2553; Eur. J. Appl. Math. 2001. V. 12. Р. 571-599; П. И. Этингоф. ДАН СССР. 1990. Т. 313. С. 42-47.

[3] S. Howison. Eur. J. Appl. Math. 1992. V. 3. P. 209-224.

[4] А. Н. Варченко, П. И. Этингоф. Почему граница круглой капли превращается в инверсный образ эллипса?. М.: Наука, 1995.

[5] Л. А. Галин. ДАН СССР. 1945. Т. 47. С. 250-253; П. Я. Полубаринова-Кочина. ДАН CCCP. 1945. T. 47. C. 254-257.

[6] B. Shraiman, D. Bensimon. Phys. Rev. A. 1984. V. 30. P. 2840-2842.

[7] M. Mineev-Weinstein, S. P. Dawson. Phys. Rev. E. 1994. V. 50. P. R24-R27; S. P. Dawson, M. Mineev-Weinstein. Physica D. 1994. V. 73. P. 373-387.

[8] M. Mineev-Weinstein, P. Wiegmann, A. Zabrodin. Phys. Rev. Lett. 2000. V. 84. P. 5106-5109.

[9] I. Krichever, M. Mineev-Weinstein, P. Wiegmann, A. Zabrodin. Laplacian growth and Whitham equations soliton theory. nlin.SI/0311005.

[10] И. М. Кричевер. Функц. анализ и его прилож. 1988. Т. 22. № 3. С. 37-52; УМН. 1989. T. 44. № 2. C. 121-184.

[11] I. Krichever. Commun. Pure. Appl. Math. 1992. V. 47. P. 437-476.

[12] С. П. Царев. ДАН СССР. 1985. Т. 282. С. 534-537.

[13] М. Шиффер, Д. Спенсер. Функционалы на конечных римановых поверхностях. М.: ИЛ, 1957.

[14] J. Hadamard. Mém. présentés par divers savants à l'Acad. sci. 1908. V. 33; П. Леви. Конкретные проблемы функционального анализа. М.: ИЛ, 1960.

[15] G. B. Whitham. Linear and nonlinear waves. New York: Wiley, 1974; H. Flashka, M. Forest, D. McLaughlin. Commun. Pure Appl. Math. 1980. V. 33. P. 739-784.

[16] P. J. Davis. The Schwarz function and its applications. The Carus Math. Monographs. № 17. Washington: The Math. Assotiation of America, 1974.

[17] B. Gustafsson. Acta Appl. Math. 1983. V. 1. P. 209-240.

[18] R. Teodorescu, E. Bettelheim, O. Agam, A. Zabrodin, P. Wiegmann. Normal random matrix ensemble as a growth problem. hep-th/0401165.

[19] V. Kazakov, A. Marshakov. J. Phys. A. 2003. V. 36. P. 4629-4640. 